\title{
L'homme au-delà de l'essence : La question de l'homme et la phénoménologie. Essai sur un écrit polémique de Husserl
}

\author{
Par UMUT ÖKSÜZAN \\ Université Galatasaray
}

\section{Introduction}

La question de l'homme est une question inévitable qui traverse toute l'histoire de la pensée. Question inévitable en ce sens précis que l'homme ne peut éviter de se mettre en question à chaque fois qu'il se découvre comme le lieu d'avènement de toute question et celui de recherche de toute réponse. Il en est toujours ainsi lors même qu'il préfère omettre cette question pour se consacrer entièrement à une autre préoccupation, ou qu'il estime nécessaire de suspendre ou de reporter son élaboration en promettant de la reprendre plus tard afin de traiter préalablement une question qui paraît être « fondamentale » ou «plus importante » à plusieurs égards et pour diverses raisons. Ce deuxième type d'omission de la question de l'homme est justifié et légitimé non seulement par la philosophie moderne, mais aussi par les sciences de l'homme et les sciences sociales comme une exigence de rigueur à respecter pour diriger la recherche selon une certaine idée de science. La question de l'homme s'efface ainsi derrière les grands débats épistémologiques et méthodologiques qui trouvent leur origine dans le souci de vérité comprise désormais comme certitude et validité universelle et objective du jugement. Ces débats arrivent, au début $\mathrm{du} \mathrm{XX}^{\mathrm{e}}$ siècle, à leur point culminant dans la phénoménologie transcendantale d'Edmund Husserl. En s'appuyant sur la radicalisation du principe de la métaphysique cartésienne par la mise entre parenthèse de la thèse de l'existence du monde, le dévoilement de la structure de la conscience et l'introduction de l'idée d'intuition des essences, 
la phénoménologie transcendantale revendique le statut de la science des sciences, du fondement de toutes les sciences possibles, y compris la philosophie. Husserl, qui tente de démontrer dans un premier temps la primauté de la phénoménologie transcendantale par la critique de toute tentative de fonder les disciplines philosophiques, comme la logique et la théorie de la connaissance, sur la psychologie expérimentale, finit par reprocher à Martin Heidegger, son assistant à l'Université de Fribourg, d'avoir dénaturé la phénoménologie par l'orientation anthropologique de son projet d'ontologie fondamentale, laquelle n'est à ses yeux rien d'autre qu'une autre forme du naturalisme, donc du relativisme et du scepticisme. En ce sens, la lecture husserlienne d'Etre et temps est d'une importance capitale pour toute réflexion critique qui cherche à répondre aux questions suivantes: est-il possible de philosopher sans prendre l'homme pour le point de départ et le point d'arrivée du questionnement ? Est-ce que les recherches philosophiques qui accordent un tel privilège à l'homme doivent être nécessairement dépréciées par la catégorie d'anthropologisme ? Est-il possible d'épuiser la question « qui est l'homme ? » par une idée de science mise au point grâce à des recherches portant sur les conditions de possibilité de la science? Les concepts et les expressions auxquels on a fait recours tout au long de l'histoire de la pensée afin de déterminer l'être, l'essence, l'idée et le qui de l'homme sont assez nombreux : "la mesure de toute chose », " animal doué de $\log o s »$, « un être créé à l'image de Dieu», «l'âme », «le corps », "l'union de l'âme et du corps », "res cogitans », "la personne ", " l'esprit », « la vie », « l'inconscient », «Dasein », « le berger de l'être ». Toutefois il parait qu'il est aujourd'hui urgent, peut-être plus urgent que jamais, de soumettre ces concepts et ces expressions à un réexamen critique pour pouvoir décider s'ils peuvent toujours nous aider à accueillir ce qui est en train d'arriver ou si nous devons commencer à penser autrement pour devenir enfin accueillant. C'est donc précisément le destin de la pensée qui se joue dans la question « qui est l'homme? ».

\section{Encore une autre attitude pseudo-scientifique : l'« anthropologisme »}

Il suffit de jeter un coup d'œil rapide sur les écrits d'Edmund Husserl pour remarquer qu'il a entièrement consacré ses efforts intellectuels à la réalisation de l'idée d'une science rigoureusement subjective et universelle. De son premier important ouvrage, publié en 1900-1901 sous le titre de Recherches ogiques, jusqu'à ses derniers écrits, notamment celui qui a été publié en 1936 à Belgrade dans la revue Philosophia et intitulé La Crise des sciences 
européennes et la phénoménologie transcendantale, Husserl n'a cessé de développer ce projet d'une science universelle et de le défendre contre les critiques et les mécompréhensions qu'il a engendré en raison de son caractère radical et audacieux dans les milieux philosophiques de l'époque ${ }^{1}$. Parmi les écrits polémiques de Husserl, celui qui porte le titre «Phénoménologie et Anthropologie» - un texte provenant des conférences qui ont été prononcées par Husserl les $1^{\text {er }}, 10$ et 16 juin 1931 à Francfort, Berlin et Halle et qui donnent non seulement un aperçu de la phénoménologie husserlienne dans son ensemble, mais aussi de multiples renseignements très utiles à propos du questionnement husserlien de la possibilité de l'anthropologie philosophique - présente un intérêt particulier pour l'investigation que nous nous donnons pour tâche de déployer en vue de répondre aux questions directrices suivantes $^{2}$ : (1) est-ce que la question «qui est l'homme ? » a un sens pour la phénoménologie transcendantale de Husserl, qui a l'ambition de découvrir et instituer le cogito, le «je pense », la «sphère de la subjectivité transcendantale » comme le lieu des évidences apodictiques et absolues sur lequel les sciences, y compris la philosophie, devraient appuyer la prétention à la validité de leurs jugements ? (2) Quel peut être le sens de la question « qui est l'homme? ? pour la réalisation d'une philosophie transcendantale ? René Descartes avec les Méditations métaphysiques, Immanuel Kant avec la Critique de la raison pure et Martin Heidegger avec le projet d'ontologie fondamentale - auquel Husserl, dans les conférences de 1931, adresse la critique de rester enfermé dans l'attitude naïve ou naturelle - s'imposent comme des interlocuteurs privilégiés à l'investigation que nous allons poursuivre en raison de son thème et de son orientation principale ${ }^{3}$.

La conférence de 1931 s'ouvre par une évaluation générale des travaux philosophiques qui ont été effectués dans les années 1920 en Allemagne et pour lesquels Husserl ne tarde pas à exprimer un avis défavorable : non seulement quant aux thèses fondamentales formulées, mais également à

${ }^{1}$ Edmund Husserl, Recherches logiques, trad. fr. H. Elie, A.L. Kelkel, R. Schérer, Paris, PUF, 1994 ; Id., La Crise des sciences européennes et la phénoménologie transcendantale, trad. fr. G. Granel, Paris, Éditions Gallimard, 1976.

2 Edmund Husserl, "Phénoménologie et Anthropologie», dans Id., Notes sur Heidegger, trad. fr. N. Depraz, D. Franck, J.-L. Fidel, J.-F. Courtine, Paris, Éditions de Minuit, 1994.

${ }^{3}$ René Descartes, Méditations métaphysiques, trad. fr. F. Khodoss, Paris, PUF, $1988^{2}$; Immanuel Kant, Critique de la raison pure, trad. fr. A. Tremesaygues et B. Pacaud, Paris, PUF, $2001^{6}$; Martin Heidegger, Etre et Temps, trad. fr. F. Vézin, Paris, Éditions Gallimard, 1986. 
propos de la démarche adoptée dans ces travaux ${ }^{1}$. En effet, on remarque que, à cette époque, les jeunes philosophes se tournent vers l'anthropologie philosophique sous l'influence de la philosophie de la vie de Dilthey. Or ce n'est là, aux yeux de Husserl, rien d'autre qu'une nouvelle figure de l'anthropologie. Mais, à en croire ce dernier, ce qui est encore plus grave pour l'avenir de la philosophie scientifique, c'est que cette influence de l'anthropologie philosophique s'étende aussi parmi les phénoménologues. Toutefois, cette critique ne vise pas uniquement les travaux des jeunes philosophes allemands, Husserl vise en premier lieu le projet de l'ontologie fondamentale - quoique sans jamais citer le nom de Heidegger. En quelques mots, il reproche à celui-ci d'avoir tenté de réformer la phénoménologie transcendantale en une « doctrine de l'essence du Dasein mondano-concret de l'homme » dans l'intention de donner un fondement à la philosophie. Husserl voit dans cette tentative heideggérienne de réformation ou de réorientation « un retournement complet de la prise de position principielle » propre à la phénoménologie transcendantale, une position qui s'oppose fermement, avec son aspiration à constituer le fondement d'une philosophie scientifique, à l'orientation subjectiviste de la pensée moderne, c'est-à-dire aux tentatives de fondation de la philosophie sur la psychologie ou l'anthropologie. En d'autres termes, l'ontologie fondamentale de Heidegger — qui se donne effectivement pour tâche de mener à bien l'élucidation du sens de l'être en général dans le cadre d'une « analytique existentiale du Dasein »est interprétée par Husserl dans la conférence de 1931 comme le strict contraire de la phénoménologie transcendantale qui veut constituer une science de la subjectivité transcendantale, une «science absolument subjective ». Cette science est en ce sens, pour Husserl, une science fondamentale récemment découverte par lui-même ${ }^{2}$. Nous essaierons de développer plus loin une analyse détaillée pour tenter de montrer que Husserl ne se contente pas de ces remarques générales sur l'ontologie heideggérienne, mais qu'il s'explique tout au long de son exposé avec les thèses fondamentales de celleci tout en maintenant son attention sur le thème de l'être du monde.

Ce qui qui nous importe ici est d'insister sur le fait que Husserl profite de l'analytique existentiale du Dasein comme d'une occasion pour accomplir une tâche qui lui parait beaucoup plus importante, celle de mettre fin au conflit entre l'anthropologisme et le transcendantalisme, un conflit qui ne

${ }^{1}$ Edmund Husserl, «Phénoménologie et Anthropologie », dans Id., Notes sur Heidegger, op. cit., p. 57-58.

${ }^{2}$ Edmund Husserl, Méditations cartésiennes, trad. fr. G. Peiffer et E. Levinas, Paris, Librairie philosophique J. Vrin, 2001, § 13, p. 60. 
recouvre rien de moins que celui entre les deux orientations subjectivistes de la pensée moderne. On sait en effet, depuis les Idées directrices pour une phénoménologie et une philosophie pures, qu'en ce qui concerne ce conflit de la modernité, qui encore aujourd'hui continue à faire sentir ses effets, la «décision de principe » doit être, selon Husserl, en faveur de la pensée transcendantale, le transcendantalisme étant la seule démarche qui se conforme à l'essence de la philosophie et qui est exigée par le sens de la tâche philosophique ${ }^{1}$. En tant qu'exigence d'une science rigoureuse, le transcendantalisme se fonde dans les évidences apodictiques, absolues et primordiales qui relèvent de l'essence même de la subjectivité transcendantale. Husserl prétend que ces évidences apodictiques ont déjà été rendues accessibles grâce à la phénoménologie constitutive ${ }^{2}$. La question de la possibilité de la fondation de la philosophie sur une anthropologie ne pourra donc se décider qu'à partir de ces évidences, qui sont, toujours aux yeux de Husserl, constamment disponibles. Bref, on peut dire que le débat engagé par Husserl contre Heidegger tourne autour des problèmes de la détermination de l'essence de la philosophie, de la tâche de la philosophie et de la méthode philosophique. Tout en admettant l'importance de la problématique de l'être pour la philosophie et la nécessité de l'élaboration des ontologies régionales, Husserl renonce néanmoins à toute tentative qui accorde une primauté à l'ontologie sur la phénoménologie transcendantale ou, en d'autres termes, une primauté à la question du sens de l'être en général au détriment de la thématique de l'ego transcendantal. Husserl voit dans l'ontologie fondamentale de Heidegger un danger qui menace la phénoménologie transcendantale, c'est-à-dire le projet de la fondation de la philosophie comme science rigoureuse, tandis qu'il caractérise lui-même, et à plusieurs reprises, la subjectivité transcendantale ou l'ego transcendantal mis à découvert par la réduction phénoménologique transcendantale comme un « domaine d'être en soi antérieur » au monde ou encore comme une " sphère nouvelle et infinie de l'existence en soi » qui, jusqu'alors, n'avait jamais été remarqués ${ }^{3}$.

On peut dès lors se poser la question de savoir pourquoi Husserl, qui en 1929 à la fin de la IV Méditation cartésienne, considère «le sens

\footnotetext{
${ }^{1}$ Edmund Husserl, Idées directrices pour une phénoménologie et une philosophie pures, trad. fr. P. Ricœur, Paris, Éditions Gallimard, 1950, § 86, p. 297-298 et § 97, p. 340-341. Cf. également Edmund Husserl, «Phénoménologie (Première version) », dans Id., Notes sur Heidegger, op. cit., p. 85-88.

2 Edmund Husserl, "Phénoménologie et Anthropologie», dans Id., Notes sur Heidegger, op. cit., p. 58.

${ }^{3}$ Edmund Husserl, Méditations cartésiennes, op. cit., § 7, p. 42, § 8, p. 47 et § 12, p. 57.
} 
authentique et universel de l'être en général et de ses structures universelles » comme l'un des problèmes constitutifs dont devrait traiter dans le concret une "philosophie phénoménologique » entendue comme "science philosophique des faits », adresse en 1931 une critique sévère à l'ontologie fondamentale de Heidegger, un travail ontologique qui semble respecter le programme de recherches fixé par Husserl lui-même ${ }^{1}$. Il est possible de dégager plusieurs renseignements utiles de l'interprétation husserlienne des philosophies pré- et post-cartésiennes, ainsi que de son explication de la phénoménologie transcendantale. Ces renseignements pourraient nous permettre de lancer une investigation ayant pour objectif de résoudre le problème de la possibilité d'une anthropologie philosophique.

\section{2. «Un radicalisme scientifique indépassable » : la phénoménologie transcendantale}

Créée par les Anciens Grecs, la philosophie est la science fondamentale en ce sens précis qu'elle prend pour thème la totalité de l'étant. D'après Husserl, cette conception de la philosophie, qui se développe progressivement au cours de son histoire, est toujours indispensable du fait qu'elle permet de rassembler toutes les sciences ${ }^{2}$. En effet, la philosophie considérée comme science de l'étant en sa totalité se distingue des autres sciences particulières en ceci que, contrairement à ces dernières, qui opèrent par des descriptions, inductions et classifications et qui, pour cette raison, ne peuvent découvrir que des "vérités relatives" ou des «vérités de situation », la philosophie aspire aux vérités apodictiques, absolues et définitives ${ }^{3}$. Pour les premiers philosophes grecs, le monde se donne de manière évidente et comme effectivement existant dans une intuition empirique, au sein d'une expérience de type pré-scientifique. Cependant, dès le début de la pensée philosophique, les propriétés du monde sont envisagées comme des propriétés en soi dont l'appréhension n'est possible que par le dépassement des limites de l'expérience. Husserl précise qu'afin d'atteindre ces propriétés en soi du monde, la philosophie, dès ses débuts, s'est tournée vers l'« eidos » ou vers le « pur a priori communément accessible dans une évidence apodictique $»^{4}$.

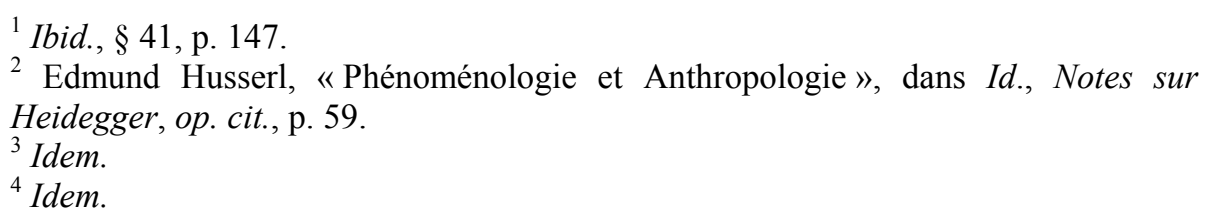


Entre parenthèses, on notera que la théorie husserlienne de l'intuition donatrice originaire, qui a la prétention de rendre accessible les essences, se situe dans le prolongement de cette ancienne solution socratique et platonicienne, et qu'elle contribue, en le radicalisant par une interrogation d'ordre transcendantal, à la continuation de cet ancien rationalisme $\mathrm{au} \mathrm{XX}^{\mathrm{e}}$ siècle. Ceci dit, il est facile de remarquer que dans cette lecture rapide et globale portant sur la genèse du rationalisme, Husserl ne tarde pas à adresser implicitement une première critique à Etre et Temps de Heidegger. De fait, la subordination de la sensation à l'idée, de l'intuition empirique à la forme invariante apriorique universelle, du monde factuel à la "pure ratio $d u$ monde » ou encore, en termes proprement husserliens, aux "possibilités d'essence », s'interprète comme la genèse d'une ontologie universelle ${ }^{1}$. Mais Husserl ajoute aussitôt que cette ontologie universelle n'est pas « seulement abstraitement générale, mais concrètement régionale ». Il nous faudra revenir sur cette proposition de Husserl, qui nous permettra de mieux comprendre l'interprétation qu'il donne du changement de la problématique philosophique à l'époque moderne et plus précisément dans les Méditations métaphysiques de Descartes.

Pour atteindre à la participation de l'« empirie » impure et «aveugle » à la « ratio » pure et clairvoyante ou, ce qui revient au même, pour parvenir à la rationalisation de l'«empirie » par le biais d'une approche méthodiquement élaborée, la connaissance rigoureusement scientifique exige que l'on accorde primauté et priorité à l'idée sur la sensation, à la connaissance rationnelle sur la connaissance des faits ${ }^{2}$. La genèse d'une «science mathématique de la nature », que Husserl étudiera plus tard en détail dans la Krisis en se focalisant sur la physique galiléenne, marque une étape décisive dans ce processus de rationalisation de la connaissance ${ }^{3}$. C'est ce qui démarque la science moderne de la philosophie antique qui, quant à elle et en dépit de tous ses efforts pour donner un caractère rationnel à la connaissance du monde, a néanmoins échoué, selon Husserl, à établir une distinction suffisamment nette entre le formel et le matériel. Ainsi, les concepts fondamentaux de la philosophie antique, tels que les concepts de «tout de l'étant» (on katalou koinon), de l'étant (to on) et de la chose (to pragma), n'étaient pas encore purement formels : ils actualisaient un sens matériel

\footnotetext{
${ }^{1}$ Idem.

${ }^{2}$ Ibid., p. 60.

${ }^{3}$ Cf. Edmund Husserl, La Crise des sciences européennes et la phénoménologie transcendantale, op. cit., § 8-12, p. 25-78.
} 
procédant de «l'étant mondain ou réal», c'est-à-dire du monde factuel ${ }^{1}$. Cette présence de la « réalité » dans des concepts qui devraient être purement formels continuera à hanter la pensée philosophique de l'Antiquité au Moyen-Âge. Ce n'est que grâce à la métaphysique de Descartes, qui a tenté de délivrer la pensée philosophique du joug de la réalité pour lui donner un nouvel élan, que, selon Husserl, le commencement d'une nouvelle ère de la science européenne peut enfin débuter ${ }^{2}$. Mais est-ce tout à fait le cas ? Ce commencement en fut-il authentiquement un ?

Malgré la découverte révolutionnaire qu'elle réalise, c'est-à-dire la découverte de l'évidence apodictique du cogito, les Méditations métaphysiques de Descartes ne réussiront pas à fonder la philosophie comme science rigoureuse. Les raisons de cet échec seront exposées par Husserl en 1929, lors de ses célèbres Conférences de Paris. L'indication de ces raisons lui permettra alors de tirer au clair la différence principielle entre la métaphysique cartésienne et la phénoménologie transcendantale. Quelles sont ces raisons ? En quoi réside cette différence de principe entre les philosophies de Descartes et de Husserl?

Dans la II $^{\mathrm{e}}$ Méditation cartésienne, plus précisément à la fin $\mathrm{du} \S 13$, Husserl reproche à Descartes de ne pas avoir donné les développements scientifiques nécessaires à l'évidence apodictique du cogito et d'avoir laissé cette évidence dans un état stérile, voire improductif ${ }^{3}$. Si Descartes n'a pas réussi à édifier une philosophie transcendantale au sens propre du terme, cet échec serait précisément dû, d'après Husserl, à ce manque de développements, qui traduit tout simplement une compréhension insuffisante de la scientificité. Husserl va donc reprendre le projet de l'élaboration d'une philosophie scientifique là où Descartes l'avait abandonné. Pour remédier à l'insuffisance scientifique de la pensée cartésienne et pour mener le projet traditionnel du rationalisme à son achèvement, il faut selon Husserl assumer et réaliser trois tâches ${ }^{4}$. (1) En premier lieu, il faut élucider l'épochè ou la réduction phénoménologique transcendantale, c'est-à-dire la méthode qui permet à l'ego méditant d'arracher son regard, son attention aux faits pour l'orienter vers les essences et, ce faisant, de passer de l'attitude naturelle à l'attitude phénoménologique transcendantale. (2) En deuxième lieu, il est nécessaire de prendre conscience du fait que l'ego réduit, l'ego transcen-

1 Edmund Husserl, «Phénoménologie et Anthropologie», dans Id., Notes sur Heidegger, op. cit., p. 60.

${ }^{2}$ Ibid., p. 60-63.

${ }^{3}$ Edmund Husserl, Méditations cartésiennes, op. cit., § 13, p. 62.

${ }^{4}$ Idem. 
dantal « constitue un champ d'investigation possible, particulier et propre». (3) En troisième et dernier lieu, il faut montrer que l'expérience que l'ego transcendantal fait de lui-même lui permet de "s'expliciter lui-même indéfiniment et systématiquement $»{ }^{1}$.

Si les deux premières tâches qui viennent d'être expliquées ont été accomplies par Husserl lui-même dès 1913, dans le premier volume des Idées directrices, la troisième tâche, qui concerne directement l'exploration de la sphère de l'ego transcendantal, possède, quant à elle, un caractère infini. Ce sont donc les futurs phénoménologues, toujours déjà endettés à l'égard des immenses travaux de Husserl, qui sont appelés à prendre aussi en charge cette tâche essentiellement infinie. En fait, l'inachèvement, comme l'affirme Husserl en 1911 dans son article intitulé la "Philosophie comme science rigoureuse », constitue le caractère essentiel de toutes les sciences qui se meuvent toujours nécessairement dans un "horizon infini de problèmes » théoriques et méthodologiques ${ }^{2}$.

Un parallèle avec Kant ne serait pas sans intérêt ici et permettrait de préciser ce qu'il s'agit d'entendre par le caractère infini de cette tâche d'autoexplicitation de l'ego transcendantal. Kant défend en effet, dans les Prolégomènes à toute métaphysique future qui pourra se présenter comme science, la thèse inverse. Comme cela est bien connu, il y caractérise la logique comme une science achevée et parfaite à laquelle on ne peut rien ajouter et dont on ne peut rien retrancher ${ }^{3}$. La critique de la raison pure est déterminée, dans le même ouvrage, de manière analogue, comme un système achevé qui servirait de fondement, dans l'avenir, à toutes les tentatives d'élaborer une métaphysique scientifique. Il faut cependant souligner que, dans l'optique de Husserl, le caractère infini de cette tâche relève de la portée infinie de la sphère de l'ego transcendantal qui, outre un «noyau d'expériences» apodictiques et adéquates, un «noyau » qui est explicité par Husserl luimême comme la présence vivante de l'ego à lui-même, comprend également un « halo » ou un « horizon » ouvert, indéterminé et vaguement général de la pensée transcendantale signifiante ${ }^{4}$. C'est à cet horizon ouvert qu'appartiennent selon Husserl les «facultés transcendantales propres du moi ». Cependant, contrairement au noyau constitué par les expériences de l'évi-

\footnotetext{
${ }^{1}$ Idem.

${ }^{2}$ Edmund Husserl, La Philosophie comme science rigoureuse, trad. fr. M. B. de Launay, Paris, PUF, $1993^{2}$, p. 13.

${ }^{3}$ Immanuel Kant, Prolégomènes à toute métaphysique future qui pourra se présenter comme science, trad. fr. M. Brunschvicg et alii, Paris, Hachette, 1891, p. 12-17.

${ }^{4}$ Edmund Husserl, Méditations cartésiennes, op. cit., § 9, p. 49.
} 
dence apodictique du sum qu'ils accompagnent chaque fois nécessairement, les actes de pensée transcendantale sont dépourvus d'évidence apodictique. C'est la raison pour laquelle ils doivent toujours être tenus sous la surveillance d'une critique qui a le pouvoir de décider s'ils sont « remplis » ou non par une « intuition correspondante » ${ }^{1}$. Dans le $\S 13$ de la $\mathrm{II}^{\mathrm{e}}$ Méditation cartésienne, Husserl reporte à une date ultérieure la réalisation d'une autre critique, à savoir celle des "principes apodictiques» de l'expérience transcendantale. Or, spécifie-t-il, tant que cette critique ne sera pas mise en œuvre, l'ego s'abandonnera aux évidences apodictiques transcendantales comme il s'abandonne dans l'attitude naturelle aux évidences des données sensibles. C'est dans le but d'éviter une telle attitude de la part de l'ego que Husserl mettra l'accent sur la nécessité d'accomplir une troisième critique, dont la tâche consistera à déterminer la portée et la validité de la connaissance transcendantale. C'est uniquement par l'accomplissement de cette troisième et dernière critique que la phénoménologie pourra gagner un statut de science mûre. Dans le cas contraire, en l'absence d'une délimitation de la connaissance transcendantale en général, la phénoménologie restera une simple description des évidences apodictiques transcendantales et ne pourra pas mettre en évidence le fondement sur lequel doit s'élever une philosophie transcendantale ${ }^{2} . \mathrm{Si}$, au premier regard, cette démarche critique de la phénoménologie husserlienne semble renouer avec les intentions de la critique kantienne, on comprend mieux, à présent, la divergence principielle entre la démarche critique de la phénoménologie et la démarche critique kantienne ${ }^{3}$.

Avant de revenir à la conférence de 1931 «Phénoménologie et Anthropologie ", il convient de dégager des Méditations cartésiennes quelques données importantes et nécessaires à l'élaboration de la question directrice de notre recherche. Dans le dernier paragraphe de la $\mathrm{I}^{\text {ère }}$ Méditation cartésienne (§11), Husserl évoquait un «fait significatif» dont on ne peut prendre conscience qu'au terme de la réduction transcendantale. En dépit de la mise entre parenthèses de la croyance à l'existence du monde qui est inhérente à l'attitude naturelle, un certain moi et sa vie psychique «demeurent intacts » et résistent à la réduction transcendantale ${ }^{4}$. Cependant, ainsi que l'affirme

\footnotetext{
${ }^{1}$ Ibid., $\S 9$, p. 50.

${ }^{2}$ Ibid., § 13, p. 60.

${ }^{3}$ Nous ne pouvons pas ici traiter de manière détaillée cette divergence qui exige de mener une étude comparative de grande ampleur. Nous nous contenterons seulement de renvoyer sur ce sujet à l'excellent article de Paul Ricœur, «Kant et Husserl », dans Kant-Studien, $\mathrm{n}^{\circ}$ 46, 1954/1955, p. 44-67.

${ }^{4}$ Edmund Husserl, Méditations cartésiennes, op. cit., § 11, p. 52-55.
}

355 
Husserl, ce moi et sa vie psychique ne font pas partie du monde. Autrement dit, Husserl leur refuse la possibilité de constituer une réalité mondaine. Il faut néanmoins insister sur le fait que Husserl leur attribue un caractère d'être, en l'occurrence un être irréel compris comme existence en soi. Au sujet de l'être irréel de ce moi et de sa vie psychique, Husserl écrit:

Et si ce moi dit : je suis, Ego cogito, cela ne veut pas dire : Je, en tant que cet homme, suis. «Moi», ce n'est plus l'homme qui se saisit dans l'intuition naturelle de soi en tant qu'homme naturel, ni encore l'homme qui, limité par abstraction aux données pures de l'expérience « interne » et purement psychologique, saisit son propre mens sive animus sive intellectus, ni même l'âme elle-même prise séparément ${ }^{1}$.

Il est à noter que, dans ce passage, nous avons affaire à un moi qui parle de lui-même. En fait, il n'y est pas question d'un moi qui dit de lui-même qu'il est ceci et qu'il n'est pas cela : il s'agit bien plutôt d'un moi qui tend vers luimême, c'est-à-dire un moi qui signifie quelque chose, qui veut dire quelque chose à propos de quelque chose. Qu'est-ce que ce moi veut dire de luimême ? Il dit de lui-même simplement ceci : ego sum, ego cogito; je suis, je pense. Nous voyons Husserl s'employer ensuite à expliquer comment il faut interpréter cette proposition « je suis », cette proposition que le moi énonce ou bien conçoit tout simplement. Le moi dit de lui-même seulement qu'il est et qu'il pense. Le moi ne dit pas explicitement ni qui il est ni ce qu'il est. Pourtant le sujet du discours, le sujet de la méditation, c'est-à-dire celui qui parle, écrit, médite ou conçoit ne s'abstient pas de se nommer, de se donner à lui-même un nom, en l'occurrence ego, le Je. L'auteur, le narrateur et la narration s'identifient dans ce nom, dans cet acte de dénomination.

L'interprétation que nous venons de donner de ce passage prend le risque d'aller à l'encontre de l'intention de Descartes avec qui, comme nous le verrons, Husserl n'est pas non plus entièrement d'accord pour des raisons phénoménologiques et transcendantales. Toutefois, outre l'explication de ce désaccord entre Descartes et Husserl, nous allons aussi essayer de montrer que notre interprétation ne s'accorde pas non plus avec l'intention de Husserl. Cette double explicitation nous permettra de revenir au problème du sens de la question anthropologique pour la phénoménologie transcendantale.

Dans la II $^{\mathrm{e}}$ Méditation métaphysique, Descartes ne s'emploie à déterminer l'essence du moi qu'après avoir démontré, par la neutralisation de l'hypothèse du malin génie, la certitude de son existence. Pour ce qui est de

${ }^{1}$ Ibid., § 11, p. 53. 
l'ordre des démonstrations cartésiennes, il suffit de rappeler ce passage de la II ${ }^{\mathrm{e}}$ Méditation:

Enfin il faut conclure, et tenir pour constant que cette proposition : Je suis, J'existe, est nécessairement vraie, toutes les fois que je la prononce ou que je la conçois en mon esprit. Mais je ne connais pas encore assez clairement ce que je suis, moi qui suis certain que je suis ${ }^{1}$

Quant à la détermination de l'essence du moi, Descartes, en prenant ses distances avec ses anciennes opinions, raisonne comme suit :

Qu'est-ce donc que j'ai cru être ci-devant? Sans difficulté, j'ai pensé que j'étais un homme. Mais qu'est-ce qu'un homme ? Dirai-je que c'est un animal raisonnable? Non, certes : car il me faudrait par après rechercher ce que c'est qu'un animal, et ce que c'est que raisonnable, et ainsi d'une seule question nous tomberions insensiblement en une infinité d'autres plus difficiles et embarrassées, et je ne voudrais pas abuser du peu de temps et de loisir qui me reste, en l'employant à démêler de semblables subtilités. Mais je m'arrêterai plutôt à considérer ici les pensées qui naissent ci-devant d'elles-mêmes en mon esprit, et qui ne m'étaient inspirées que de ma seule nature, lorsque je m'appliquais à la considération de mon être ${ }^{2}$.

C'est justement à ce passage de la $\mathrm{II}^{\mathrm{e}}$ Méditation métaphysique que Husserl fait allusion quand il affirme que l'ego n'est pas l'homme. Il est facile de remarquer que Husserl pense toujours dans le sillage du rationalisme cartésien quand il affirme que le moi n'est pas l'homme qui se saisit luimême dans l'intuition naturelle de soi en tant qu'homme naturel. Ce que le doute méthodique de Descartes met entre parenthèses, met hors valeur afin de parvenir à l'évidence apodictique fondamentale, à la clara et distincta perceptio, c'est précisément les données sensibles dans leur totalité. En répétant le geste de Descartes, Husserl participe, du moins dans ces lignes, à la dévalorisation philosophique de la thèse aristotélicienne "l'homme est un animal doué de logos ». Ainsi l'animal et l'homme comme une espèce d'animal sont-ils expulsés de la problématique de la philosophie. Toutefois, il est à souligner que cette expulsion, définitive dans la métaphysique cartésienne, est provisoire dans la phénoménologie husserlienne, tout simplement parce qu'elle promet d'expliciter l'intentionnalité constituante, c'est-à-dire qu'elle se donne pour tâche d'expliquer comment l'ego se constitue comme

${ }^{1}$ René Descartes, Méditations métaphysiques, op. cit., p. 39.

${ }^{2}$ Ibid., p. 39. 
« existence en soi » et comment il constitue en lui l'autre moi, l'alter ego et le non-moi.

Cependant, à propos de la détermination husserlienne de l'ego, il faut aussi indiquer que, si Husserl entend par « intuition naturelle » ce que Kant a désigné sous le terme d'«intuition sensible», le fait qu'il emploie une expression telle qu' " intuition naturelle de soi » pour accentuer la particularité existentielle de l'ego et de la sphère égoïque donne à entendre qu'il suppose, peut-être à son insu, un rapport d'identité entre la nature et la sensibilité. La mise en examen de la possibilité d'une « intuition naturelle » exige par conséquent un retour à la conception kantienne de l'intuition.

On sait que Kant fait recours au terme «intuition » pour désigner l'intuition sensible a posteriori ou l'intuition pure a priori, et qu'il explicite l'intuition pure a priori comme la forme de l'intuition sensible ${ }^{1}$. Il faut cependant souligner que l'intuition en tant que telle, même lorsqu'elle est sensible ou a posteriori, n'est pas naturelle. Dans la critique kantienne, la nature, prise au sens matériel du terme, n'est rien d'autre qu'un simple nom destiné à signifier l'ensemble des phénomènes. Plus exactement, le terme de «nature» signifie le plus souvent l'objet qui se donne dans l'intuition sensible extérieure, c'est-à-dire l'objet qui se donne dans l'espace et dans le temps. Il est possible de constater que le terme de "nature» est aussi employé par Kant pour désigner le moi pensant qui ne peut constituer un objet que pour autant qu'il se donne dans une intuition sensible intérieure, c'est-à-dire dans le rapport du temps ${ }^{2}$. Dans la critique des paralogismes de la raison pure, une critique qui se donne pour tâche de dévoiler le caractère dogmatique de la psychologie rationnelle et les contradictions qu'elle engendre dans la raison, Kant affirme plus d'une fois qu'ego cogito, « je pense » n'est rien d'autre qu'une « proposition formelle de l'aperception » et que «cette proposition n'est pas une expérience, mais la forme de l'aperception qui est inhérente à toute expérience et qui la précède ${ }^{3}$. Bref, selon Kant, ego cogito, « je pense » est seulement la «condition subjective d'une connaissance possible en général ». Nous reviendrons plus loin à cette caractérisation fonctionnelle de l'ego cogito.

À la fin du passage dont nous venons de donner une première interprétation, Husserl déclare que l'ego n'est pas non plus l'homme qui est réduit par abstraction aux données psychologiques pures de l'expérience interne et

${ }^{1}$ Sur ce sujet, $c f$. Immanuel Kant, Critique de la raison pure, op. cit., § 8, p. 68-75.

${ }^{2} C f$. Immanuel Kant, Prolégomènes à toute métaphysique future qui pourra se présenter comme science, op. cit., §36, p. 131-132.

${ }^{3}$ Immanuel Kant, Critique de la raison pure, op. cit., p. 287. 
qui saisit, grâce à cette abstraction, son propre esprit, entendement et raison. Or c'est précisément par ces termes que Descartes détermine l'essence de l'homme quand il s'arrête à un attribut de l'âme, à savoir la pensée, après avoir éliminé d'autres attributs de l'âme ainsi que tous les attributs du corps :

Un autre est de penser, et je trouve ici que la pensée est un attribut qui m'appartient : elle seule ne peut être détachée de moi. [...] Je suis donc, précisément parlant une chose qui pense, c'est-à-dire un esprit, un entendement ou une raison, qui sont des termes dont la signification m'était auparavant inconnue $^{1}$.

Il est clair que Husserl, par l'interprétation qu'il en donne, s'écarte manifestement de la détermination cartésienne de l'essence de l'ego. Par ailleurs, nous voyons aussi Husserl reprocher à Descartes, qui prétend mettre hors jeu l'homme dans la détermination de l'essence de l'ego, de décrire l'ego par l'un des attributs de l'homme et de réifier l'ego cogito, le « je pense », la conscience. De ces considérations, nous pouvons conclure que la psychologie empirique n'est, aux yeux de Husserl, qu'une branche de l'anthropologie et que l'essence de l'ego transcendantal ne saurait être limité ni par l'acte de penser ni par le pouvoir de penser. Conclure de l'ego cogito à la res cogitans, $\mathrm{du}$ 《 je pense » à une chose qui pense consiste, selon Husserl, à retomber dans ce qui est mis entre parenthèse par le doute méthodique ou, dit autrement, dans la naïveté de l'attitude naturelle et à réaffirmer la croyance qui caractérise essentiellement l'attitude naturelle, à savoir la croyance ontologique du monde ${ }^{2}$.

La croyance ontologique du monde, comme on le sait, est la seule solution que, dans $\mathrm{la} \mathrm{VI}^{\mathrm{e}}$ Méditation métaphysique, Descartes peut proposer par manque de preuve au problème de l'existence du monde. Dans la Critique de la raison pure, Kant tente de résoudre le même problème en accordant l'idéalisme transcendantal ou critique avec le réalisme empirique sur le fondement de la distinction du phénomène et de la chose en soi, une distinction que Husserl rejette catégoriquement dans $\mathrm{la} \mathrm{IV}^{\mathrm{e}}$ Méditation cartésienne pour cette simple raison qu'elle « laisse ouverte la possibilité de choses en soi, ne fût-ce qu'à titre de concept-limite $»^{3}$. Que Husserl renonce aux réalismes cartésien et kantien montre qu'il juge nécessaire de maintenir et de poursuivre le doute méthodique dans la réduction pour accomplir, comme il le souligne dans la conférence "Phénoménologie et Anthropo-

${ }^{1}$ René Descartes, Méditations métaphysiques, op. cit., p. 41.

${ }^{2}$ Edmund Husserl, Méditations cartésiennes, op. cit., § 10, p. 50-52.

${ }^{3}$ Ibid., $\S 41$, p. 144. 
logie », un «radicalisme scientifique indépassable», et ce grâce à la découverte d'un fondement ultime et apodictiquement évident de la validité de toute connaissance ${ }^{1}$. De cette exigence de maintenir le doute, nous pouvons conclure que la phénoménologie transcendantale n'est possible que sous la forme d'un idéalisme sceptique qui est, selon Kant, une espèce de l'idéalisme empirique provenant d'une «fausse incertitude de nos perceptions extérieures $»^{2}$. C'est précisément cette incertitude qui accompagne la phénoménologie husserlienne dans toutes les étapes de sa démarche. La méfiance constante envers la vérité des données sensorielles et perceptives, qui trouve son origine dans le rationalisme platonicien, un rationalisme dont le dépassement a été préparé par Descartes et accompli ensuite par Kant, détermine l'orientation fondamentale du questionnement phénoménologique de Husserl dans sa recherche des possibilités d'essence qui seraient intuitivement appréhendées dans leur évidence apodictique et universelle et à laquelle le contenu de tout acte de conscience devrait correspondre pour qu'il puisse avoir un sens et une valeur ontologique. Il n'est pas étonnant de voir l'ego de l'ego cogito, avec sa présence vivante à lui-même, s'imposer dans l'intuitionnisme husserlien comme un généreux donateur du sens qui se montre méfiant envers toute autre donation que les siennes et qui, conscient de ce qu'il donne, laisse sa générosité se transformer en une domination. Il nous paraît possible et d'ailleurs nécessaire de considérer les efforts de Heidegger, Levinas et Derrida comme une lutte menée contre cette aspiration de la phénoménologie husserlienne à l'institution d'une domination égologique et égocentrique au nom de la scientificité.

\section{La résistance irréductible : l'être et l'être-homme}

Le choix du thème et du problème de la conférence de 1931 témoigne de la prise de conscience par Husserl de cette lutte contre l'égologie transcendantale. C'est contre l'interprétation heideggérienne du monde comme moment de la constitution fondamentale du Dasein qu'il réaffirmera la possibilité essentielle inhérente à l'ego de saisir dans une expérience transcendantale son existence en soi et son antériorité en soi dans l'épochè du monde. C'est en ce sens que l'épochè s'interprète comme une «véritable

${ }^{1}$ Edmund Husserl, «Phénoménologie et Anthropologie», dans Id., Notes sur Heidegger, op. cit., p. 62.

${ }^{2}$ Immanuel Kant, Critique de la raison pure, op. cit., p. 305. 
révolution » produite par et dans l'ego philosophant ${ }^{1}$. L'expérience transcendantale que l'ego est capable de faire de lui-même constitue, d'après Husserl, la condition de possibilité pour réussir à donner un sens au monde et à développer une «science radicalement responsable $»^{2}$. Contrairement à l'analytique existentiale de Heidegger, qui explicite les phénomènes de l'angoisse (Angst) et de la conscience (Gewissen) comme condition de possibilité de la singularisation du Dasein, Husserl affirme que c'est par la réduction que l'ego se libère de son être-réel, de son être-homme singulier parmi les hommes pour devenir un ego transcendantal. Autrement dit, Husserl reproche à Heidegger de confondre la « solitude humaine » avec la « solitude transcendantale ${ }^{3}$.

Pour mieux comprendre ce désaccord entre Husserl et Heidegger, il faut rendre compte de la manière dont ils explicitent la possibilité de saisir ce qui est fondamental. En fait, chez Husserl, l'ego naturel et l'ego transcendantal sont caractérisés comme deux possibilités contemporaines de l'ego. L'antériorité de l'ego transcendantal vis-à-vis de l'ego naturel tient à ce qu'il se constitue comme une instance ayant le pouvoir de saisir par l'intuition les évidences apodictiques universelles et de donner un sens au monde réellement saisi par l'ego naturel. Il revient aussi à l'ego transcendantal d'opérer la réduction du monde. L'ego transcendantal procède en toute liberté à la réduction en vue de se saisir lui-même comme le fondement ultime apodictiquement évident de toute connaissance possible en général. Autrement dit, la liberté de l'ego transcendantal, en tant que liberté ultime et sans fondement, s'impose comme le seul fondement, la seule raison possible de toute réduction transcendantale. Husserl précise que l'ego transcendantal est dans l'obligation de maintenir l'être du monde dans la réduction pour ne pas permettre à l'ego naturel d'orienter les opérations scientifiques dans une perspective réaliste.

À vrai dire, Heidegger reste fidèle à ce schéma phénoménologique husserlien quand il tente d'expliciter l'être authentique et l'être inauthentique du Dasein dans Être et Temps. Ces deux modes d'être du Dasein sont encore une fois interprétés comme des modes d'être possibles contemporains, et la priorité est accordée à l'être authentique, terme qui joue un rôle déterminant dans le rapport. Heidegger décrit la déchéance (Verfallen) et la publicité (Öffentlichkeit) en tant que moments de la quotidienneté manifestant une

1 Edmund Husserl, "Phénoménologie et Anthropologie», dans Id., Notes sur Heidegger, op. cit., p. 63.

${ }^{2}$ Idem.

${ }^{3}$ Ibid., p. 64. 
fuite du Dasein devant sa possibilité d'être authentique ${ }^{1}$. Cette fuite n'est ni fortuite ni sans motif, elle est inévitable et motivée par la compréhension du sens de l'être qui est toujours à l'œuvre dans l'existence. En d'autres termes, c'est parce qu'il comprend chaque fois le sens de l'être que le Dasein fuit devant lui-même et se perd dans des préoccupations quotidiennes. Le Dasein existe, selon Heidegger, le plus souvent non pas sur le mode d'être soi, mais sur le mode d'être anonyme du On (das Man $)^{2}$. Dans ce mode d'être anonyme, le Dasein comprend de manière impropre son être selon le mode d'être des choses du monde, plus précisément selon le mode d'être que Heidegger appelle Vorhandenheit.

À la différence de la théorie husserlienne de la réduction, dans l'ontologie fondamentale de Heidegger, le sens de l'être, le sens de l'être du Dasein et le sens de l'être du monde ou de la mondanéité - qui s'entendent toujours implicitement et agissent toujours à l'arrière-plan de l'existence se révèlent au Dasein d'abord dans une compréhension pré-conceptuelle, pré-scientifique et pré-ontologique. Cette compréhension ne se réalise pas par le biais d'une réduction de l'être du monde, mais par une perte momentanée du sens de l'être du monde, qui se traduit par l'interruption du déroulement habituel des préoccupations quotidiennes ${ }^{3}$. Cette interruption n'est pas l'œuvre du Dasein lui-même. Elle est la conséquence d'un événement qui survient et peut survenir à tout instant au sein du monde ambiant, du monde de la préoccupation. Heidegger soutient que cet événement peut advenir selon trois formes principales, comme ce qui vient endommager, importuner et qui ne se laisse pas faire ${ }^{4}$. Dans toutes ces formes, l'événement survenu dévoile non seulement la mondanéité du monde comme un réseau de renvois ou une significativité, mais aussi la familiarité originaire du Dasein avec le monde, c'est-à-dire l'appartenance irréductible du monde à sa constitution ontologique, désignée dans Etre et Temps par l'expression être-au-monde (In-der-Welt-sein). On remarquera également que ce dévoilement de la mondanéité et de la constitution d'être du Dasein sert de fondement à la formation de la compréhension de l'être du Dasein et à l'explicitation ontologique du sens de l'être du Dasein et du sens de l'être en général ${ }^{5}$. Bref, des analyses qui viennent d'être exposées nous pouvons conclure qu'en ce qui concerne le dévoilement du sens de l'être du fonde-

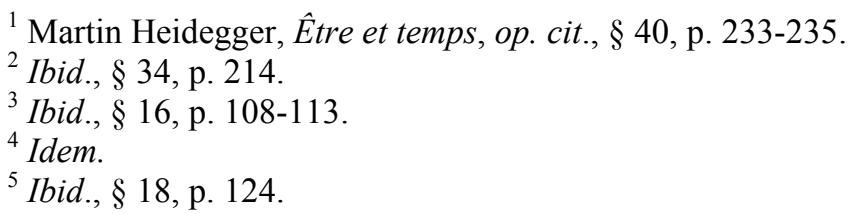


ment, Heidegger juge insoutenable, du point de vue ontologique, la réduction husserlienne : elle refuse en effet de donner une place dans la constitution ontologique de l'ego à l'apparition libre de ce qui est radicalement autre, de ce qui ne peut jamais être constitué après coup par l'ego comme un alter ego ou comme un non-moi. Le même et l'autre résident «co-originairement» et simultanément à titre de possibilités d'être dans la structure ontologique de l'étant.

Mais nous voyons Husserl s'opposer fermement en 1931 à l'explicitation heideggérienne du Dasein et de la mondanéité. La réduction transcendantale n'est qu'une étape dans la démarche phénoménologique. Elle a pour fonction de thématiser le champ de l'ego transcendantal et de rendre possible la réflexion transcendantale. Celle-ci, en focalisant à son tour l'attention sur les vécus de conscience réduits, permettrait de dévoiler, d'une part, les propriétés transcendantales de la conscience et, d'autre part, «le monde en tant que phénomène universel de validité, en tant qu'univers permanent opposé à la multiplicité de la conscience du monde ${ }^{1}{ }^{1}$. Or, un univers permanent opposé à la multiplicité de la conscience du monde, n'est-ce justement pas le rêve le plus ancien de la métaphysique? N'est-ce pas le désir d'Archimède qui déclare qu'il n'a besoin de rien d'autre que d'un point fixe pour tirer la terre de sa place? La phénoménologie husserlienne, par cette volonté d'atteindre par l'intuition éidétique à un univers permanent, fixe, immuable, absolu, ne prend-t-elle pas sa place dans la métaphysique, c'est-à-dire dans une époque de l'histoire de la pensée ?

C'est en effet la pensée qui s'impose à elle-même la découverte des points, des concepts, des règles fixes et immuables comme une exigence à satisfaire pour pouvoir rendre intelligible le monde qui apparaît toujours comme étant en changement perpétuel. C'est la pensée qui, après les avoir déduites des formes de jugements, fixe ses représentations propres comme des concepts purs de l'entendement. Il revient aussi à la pensée de s'imposer l'exigence de subsumer les représentations sensibles sous les concepts purs de l'entendement pour les synthétiser selon des règles pures a priori et constituer par ces synthèses une connaissance scientifique. C'est encore une fois la pensée qui fait une distinction entre les facultés et qui revient ensuite sur cette distinction pour la corriger ou la préciser davantage. Kant ne se plaignait-il pas de ce que l'on n'a pas pu distinguer nettement l'entendement

${ }^{1}$ Edmund Husserl, «Phénoménologie et Anthropologie», dans Id., Notes sur Heidegger, op. cit., p. 65. 
et la raison avant sa critique de la raison ${ }^{1}$ ? L'ego de l'ego cogito, insondable selon Kant, n'est-il rien d'autre qu'une invention conceptuelle de la pensée, une invention du cogitare qui cherche à fixer son être effectif, c'est-à-dire ce qu'il saisit et peut ressaisir à tout moment par la réflexion, par la pensée de la pensée? Kant soutient l'idée qu'il n'existe aucun objet qui puisse correspondre à l'aperception pure ou transcendantale. Car l'objet ne peut se présenter que comme phénomène, c'est-à-dire comme une donnée de l'intuition sensible. L'aperception transcendantale en tant qu' " acte de la spontanéité » n'a pas d'autre fonction que celle d'accompagner toutes les autres représentations et de les rassembler dans une conscience générale de $\operatorname{soi}^{2}$. Il est clair que Husserl n'est pas satisfait de cette explication kantienne de l'aperception transcendantale. Il juge nécessaire que la pensée portant sur une essence pure soit remplie par une vision de l'essence correspondante, en d'autres termes par une intuition éidétique correspondante. Même ego cogito, le « je pense », la conscience devrait être remplie et, par là même, validée par une intuition éidétique pour gagner le statut d'évidence apodictique ${ }^{3}$. La phénoménologie transcendantale deviendrait une science rigoureuse par l'explicitation de ce remplissement. C'est seulement par cette explicitation phénoménologique que l'on pourrait se protéger contre les spéculations. La conférence de 1931 travaille à consolider la radicalité du questionnement phénoménologique. La réduction transcendantale est tellement radicale qu'elle ne laisse pas en dehors de son champ d'activité la pensée effective elle-même. "Se considérer en tant qu'homme", prétendre procéder à la réduction en tant que "moi cet homme concret», tout cela revient selon Husserl à «présupposer la validité du monde » et à retomber dans l'attitude naturelle ${ }^{4}$. Ni le corps ni l'esprit ne sauraient résister à la réduction transcendantale. Cependant cela ne signifie aucunement l'abandon des questions philosophiques du monde et de l'homme. La phénoménologie qui s'éloigne en un premier temps des questions philosophiques promet d'y revenir par la suite pour leur donner un nouveau sens et une nouvelle validité. L'homme qui perd toute sa valeur dans la réduction recevra ultérieurement son sens au sein d'une « aperception universelle monde $»^{5}$. Autrement dit, c'est dans une

\footnotetext{
${ }^{1}$ Immanuel Kant, Prolégomènes à toute métaphysique future qui pourra se présenter comme science, op. cit., § 41, p. 153-154.

${ }^{2}$ Immanuel Kant, Critique de la raison pure, op. cit., p. 287-288.

${ }^{3}$ Edmund Husserl, Méditations cartésiennes, op. cit., § 30-34, p. 113-125.

4 Edmund Husserl, «Phénoménologie et Anthropologie», dans Id., Notes sur Heidegger, op. cit., p. 65.

${ }^{5}$ Idem.
} 
aperception empirique accomplie par l'ego transcendantal lui-même que le monde, la nature et le «Dasein en tant qu'homme» dans ce monde peuvent devenir un étant pour l'ego transcendantal et regagner de ce fait leur sens et leur validité d'être, en un mot leur évidence phénoménologique propre.

Pour parvenir à distinguer la réduction transcendantale d'une simple négation de la réalité, Husserl affirme ceci :

Je n'ai donc rien perdu de ce qui était là pour moi dans la naïveté et qui, en particulier, se montrait à moi comme effectivité étante. Bien plus : dans l'attitude absolue, je connais le monde lui-même et le connaît maintenant comme ce qu'il était toujours et devait être essentiellement pour moi : un phénomène transcendantal ${ }^{1}$.

L'être de l'étant, qu'il soit possible ou effectif, ne saurait donc avoir un sens que relativement à la conscience transcendantale, qui est capable de l'expliciter dans une intuition éidétique. Dans la perspective de Husserl, cette relativité transcendantale est inévitable. En ce sens, l'analytique existentiale de Heidegger n'est pas éloignée de l'égologie husserlienne. L'être qui est toujours l'être de l'étant ne peut être explicité quant à son sens que dans la compréhension (Verstehen) et la disposition (Befindlichkeit), deux structures ontologiques fondamentales de l'«être-à » du Dasein. L'être ne pourrait pas se montrer dans son sens - ou ce qui, selon Heidegger, revient au même, dans sa vérité - si le Dasein n'existait pas. Mais ce serait commettre une grave erreur qui aurait des conséquences désastreuses que d'expliciter ontologiquement le Dasein et, a fortiori l'homme, comme un étant là-devant qui se rencontrerait dans le monde ambiant et qui ne saurait recevoir un sens qu'à la suite de l'épochè de son être. En effet, la réalité peut avoir des dimensions ou des aspects qui ne sont pas encore maitrisables par une "légalité essentielle, apodictique, de la corrélation» entre le cogito et le cogitatum, c'est-à-dire l'objet intentionnel ${ }^{2}$. Heidegger s'oppose fermement à la détermination du Dasein comme un étant là-devant et adresse une critique à la phénoménologie husserlienne dans ces lignes d'Être et temps :

On peut se refuser à faire de l'âme une substance, refuser de conférer la choséité à la conscience et l'objectivité à la personne, reste que, ontologiquement,

${ }^{1}$ Ibid., p. 67.

${ }^{2}$ Ibid., p. 69. 
on persiste à appliquer, explicitement ou non, quelque chose qui garde le sens de l'être-là-devant ${ }^{1}$.

Avant de conclure, il convient de citer cet autre passage du $§ 25$ d'Etre et temps afin de mettre en évidence la différence principielle entre l'égologie husserlienne et l'analytique existentiale de Heidegger :

Va-t-il donc a priori de soi que l'accès au Dasein doive être une réflexion simplement percevante sur le je des actes? Et si cette sorte d'«auto-donation » du Dasein était pour l'analytique existentiale une fausse piste et, à vrai dire, une tentation ayant son fondement dans l'être du Dasein lui-même ? Sans doute le Dasein, sitôt qu'il se parle à lui-même, se dit-il toujours : je le suis, et finalement ne le dit-il jamais si fort que lorsqu'il n'est «pas » cet étant. Et si la constitution du Dasein, selon laquelle il est chaque fois à moi, était la raison pour laquelle d'abord et la plupart du temps le Dasein n'est pas soi-même? Et si l'analytique existentiale courait en quelque sorte à sa perte en prenant pour point de départ cet être-donné du je au Dasein même et à une auto-explicitation toute prête ? Et s'il devait en ressortir que l'horizon ontologique dans lequel se détermine ce qui est accessible par simple donation demeure indéterminé dans son principe ? On peut bien être toujours en droit de dire ontiquement de cet étant que «je» le suis. L'analytique ontologique, si elle fait usage des énoncés de ce genre, doit cependant les placer sous des réserves de principe. Le «je » doit être seulement entendu au sens d'une indication n'engageant à rien de plus, indication formelle de quelque chose qui, dans chaque contexte d'être phénoménal, peut éventuellement se révéler comme son « contraire ». Mais ici «non-je » ne se ramène alors en aucune façon à l'étant par essence dénué d' "égoïté » mais signifie, au contraire, un genre d'être déterminé du «je» lui-même, par exemple celui de s'être-soi-même-perdu ${ }^{2}$.

Le Dasein est un étant pour lequel en son être il y va de cet être même. Dans l'analytique existentiale, le sens de l'être du Dasein s'interprète comme souci (Sorge), mais il ne faut pas perdre de vue que ce n'est pas un souci de soi, mais précisément un souci qui concerne à la fois son être propre et l'être de l'autre. Il faut aussi mettre l'accent sur le fait que l'analytique existentiale contient des analyses qui ne sont pas moins transcendantales que celles développées dans la phénoménologie husserlienne. En partant des descriptions de l'existence dans la réalité quotidienne, l'analytique existentiale s'avance toujours vers l'explicitation des structures ontologiques a priori

${ }^{1}$ Martin Heidegger, Être et temps, op. cit., § 25, p. 156.

${ }^{2}$ Ibid., $\S 25$, p. 157-158. 
transcendantales du Dasein. Heidegger abandonne lui aussi, comme le fait Husserl, les concepts de la métaphysique traditionnelle tels que l'âme, le corps, l'esprit, l'intelligence, la raison, l'homme. Il emploie d'ailleurs très prudemment le terme de nature. Mais pour Heidegger, la question fondamentale de la philosophie est la question du sens de l'être en général. Heidegger parvient à la position de cette question fondamentale par la radicalisation de la question aristotélicienne de l'étant comme tel (to on he on), la question de l'être de l'étant. Cette question fondamentale en vertu de sa radicalité représente une nouvelle étape dans l'histoire de la philosophie. Elle ne peut être ni préparée ni élaborée par une phénoménologie égologique du fait qu'elle comprend aussi bien la question de l'être de la subjectivité transcendantale possible et effective que celle de la légitimité ontologique d'une phénoménologie transcendantale qui cherche à fonder le sens, la valeur et la vérité de l'être réel dans l'être irréel des essences. 